\title{
Fertilización Con Potasio Y Fosfitos, Sobre El Rendimiento De Maíz Duro (Zea Mays) En La Zona Subcentral Litoral
}

\author{
Ing. Agr. Álvaro Lamilla Arana \\ Ing. Agr. Eduardo Colina Navarrete Mg. Sc. \\ Ing. Ind. Carlos Castro Arteaga Mg. Sc. \\ Ing. Agr. Danilo Santana Aragone \\ Ing. Agr. Guillermo García Vásquez Mg. Sc. \\ Ing. Agr. Oscar Mora Castro MBA \\ Lcda. Martha Uvidia Vélez MIS \\ Ing. Agr. Joffre León Paredes MBA \\ Universidad Técnica de Babahoyo, Ecuador \\ Ing. Agr. Miguel Goyes Cabezas MBA \\ Unidad Nacional de Almacenamiento-UNA
}

Doi: 10.19044/esj.2018.v14n15p46 URL:http://dx.doi.org/10.19044/esj.2018.v14n15p46

\begin{abstract}
The investigation evaluated the effect of fertilizers potásicos with fosfitos on the yield of grain of hard corn (Zea mays L.), in the Property "Santiago", located in the Km 26 way Babahoyo-San Juan, county of Los Ríos. A rehearsal settled down in the hybrid corn DK-7088, measuring the answer from the corn to the application of fertilizers potasic and fhosphite, to determine the dose with more effect on the production. 19 treatments were proven with Chloride of potassium, Sulfate of potassium and Nitrate of potassium, with fhosphite of Calcium, Magnesium and Potassium, more a witness without applications. The used experimental design was complete Blocks at random in three repetitions, the evaluated variables were subjected to the variancia analysis, and the test was applied from Tukey to $95 \%$ of probability. The evaluated parameters were: plant height, days to flowering, longitude and ear diameter and yield for hectare. Found increases in grain yield with three treatments, highlighting the treatment with the application of muriate of potash in conjunction with Calcium phosphide $\left(9451,65 \mathrm{~kg} \mathrm{ha}^{-1}\right)$. The results indicate the possibility of increases of more than $49 \%$ in relation to the witness (6339 $\mathrm{kg} \mathrm{ha}^{-1}$ ) where there was applied the treatments.
\end{abstract}

Keywords: Fertilizer, Yield, Conventional, Corn, Biomass 


\section{Resumen}

La investigación evaluó el efecto de fertilizantes potásicos con fosfitos sobre el rendimiento de grano de maíz duro (Zea mays L.), en la Finca "Santiago", ubicada en el Km 26 vía Babahoyo-San Juan, Provincia de Los Ríos. Se estableció un ensayo en el maíz híbrido DK-7088, midiendo la respuesta del maíz a la aplicación de fertilizantes potásicos y fosfitos, para determinar la dosis con mayor efecto sobre la producción. Se probaron 19 tratamientos con Muriato de potasio, Sulfato de potasio y Nitrato de potasio, con fosfitos de Calcio, Magnesio y Potasio, más un testigo sin aplicaciones. El diseño experimental utilizado fue Bloques completos al azar en tres repeticiones, las variables evaluadas fueron sometidas al análisis de variancia, y se aplicó la prueba de Tukey al 95\% de probabilidad. Los parámetros evaluados fueron: altura de planta, días a floración, longitud y diámetro de mazorca y rendimiento por hectárea. Se encontró incrementos en el rendimiento de grano con tres tratamientos, destacándose el tratamiento con la aplicación de Muriato de potasio en conjunto con Fosfito de Calcio $(9451,65$ $\mathrm{kg} \mathrm{ha}^{-1}$ ). Los resultados indican la posibilidad de incrementos de más del $49 \%$ con relación al testigo (6339 $\left.\mathrm{kg} \mathrm{ha}^{-1}\right)$ donde no se aplicó los tratamientos.

Palabras-Claves: Fertilizante, Rendimiento, Convencional, Maíz, Biomasa

\section{Introducción}

El maíz (Zea mays L), es por hoy uno de los productos agrícolas más importante de la economía Ecuatoriana y mundial, esto debido a que casi las tres cuartas partes de la producción total proviene de unidades familiares campesinas, la mayorías de ella de economías de subsistencia, teniendo una elevada incidencia social.

El mundo siembra anualmente 129,2 millones de hectáreas aproximadamente, alcanzado una producción de alrededor de 477,4 millones de toneladas métricas. En Ecuador este cultivo es el segundo grano más importante en la alimentación humana después del arroz, empleando alrededor de 40000 personas en mano de obra, correspondiente el 11\% de la población económicamente activa dedicada a la agricultura (INEC-SINAGAP, 2014).

El maíz (Zea mays L.), es uno de los principales cultivos del país y su importancia radica en su utilización no solo como fuente de alimento para seres humanos, sino también por el uso que se le da en la elaboración de alimento balanceado. La siembra de 361347 hectáreas, se producen sobre todo en las provincias de Los Ríos, Guayas y Manabí con el 75\% del área total nacional. El promedio de producción es de 3,5 toneladas por hectárea lo cual es relativamente bajo y no abastece la demanda de la agroindustria ecuatoriana. Los Ríos cuenta con 156565 hectáreas sembradas y un 
rendimiento promedio de 4,56 toneladas por hectárea lo cual es bajo, principalmente debido al escaso uso de semillas certificadas y de alto rendimiento (MAGAP, 2016).

Los problemas de nutrición de los cultivos a través del tiempo, ha hecho que se presenten de calidad de suelo. Estos han sufrido problemas de degradación física, biológica y química. Esto se debe a la calidad de nutrientes utilizados. Las fuentes modernas de nutrientes en su mayoría son sales acidificantes que desmejoran la calidad del recurso edáfico. El mal uso de los fertilizantes potásicos por parte de los agricultores sumado a al mal manejo tecnológico que le dan al cultivo, hace que los rendimientos del cultivo de maíz sean bajos.

Melgar (2009) menciona que el potasio es esencial para el crecimiento de las plantas, las cantidades de potasio absorbidas por los cultivos son casi tan grandes como las del nitrógeno y en algunos cultivos mucho mayor (soja o banano). Las plantas absorben el potasio como ión y su función es en la mayoría de los procesos vitales de la planta. Su rol en la planta es múltiple ya que es el catión más importante, no solo respecto a su alto contenido en los frutos, sino también respecto a sus funciones bioquímicas y fisiológicas tales como: a) Activación de enzimas; b) Crecimiento y división celular en tejidos jóvenes; c) Síntesis de carbohidratos, proteínas y aceites; d) Transporte de azúcares a través del floema usando la ATP como fuente de energía; e) Uso del agua: la absorción de agua por las raíces y regulación de la transpiración; f) Mayor tolerancia a condiciones estresantes debido a la sequía, salinidad, heladas y enfermedades, y g) Regulador de los balances iónicos en la planta.

El Potasio $(\mathrm{K})$ es fundamental en el proceso de la fotosíntesis, deficiencia de $\mathrm{K}$ reduce la fotosíntesis e incrementa la respiración celular, resultando en una reducción de la acumulación de carbohidratos y por consecuencia un efecto adverso en el crecimiento y producción de la planta. Una planta bien nutrida con $\mathrm{K}$ tiene una mayor capacidad de soportar condiciones de estrés por falta o exceso de agua, esto ya que el $\mathrm{K}$ es determinante en la capacidad de los estomas de abrir y cerrar cuando la planta está sometida a condiciones de sequía (USDA, 2004).

Para el García (2013) en general la fertilización con potasio es muchas veces desatendida por el impacto indirecto del $\mathrm{K}$ en los rendimientos, y por el hecho que los cultivos pueden producirse dentro de un amplio rango de disponibilidad de $\mathrm{K}$ en el suelo a diferencia de los otros dos nutrientes $\mathrm{N}$ y $\mathrm{P}$. Eso lleva en muchos escenarios a bajas aplicaciones de potasio en un contexto de fertilización desbalanceada, lo que conduce a una disminución de las reservas de los suelos, pérdidas de rendimiento y mayor riesgo económico para los productores agrícolas. A nivel mundial, el desbalance en favor del $\mathrm{N}$ ha ido creciendo en el mediano plazo, en particular en países en desarrollo. 
Estudios realizados en la Universidad de Jerusalén (Kant, S., Kafkafi, U. 2010) indican que los granos absorben $\mathrm{K}$ del suelo generalmente antes de la etapa de llenado o incluso de la floración. El K encontrado en las espigas de maíz depende completamente de la redistribución desde otros órganos de la planta. Las flores, frutos en desarrollo y tubérculos sirven como destino o «reservorio» del K. Estos órganos movilizan al potasio desde las hojas. Si durante las primeras etapas de desarrollo de las plantas los niveles de $\mathrm{K}$ son bajos, la demanda en una fase posterior del crecimiento de los frutos puede dar lugar a deficiencias de $\mathrm{K}$ en hojas. Este agotamiento de $\mathrm{K}$ de las hojas por parte de la remobilización hacia los órganos fructíferos conduce a una reducción en su actividad fotosintética que más adelante conducirá a una menor producción o disminución de la calidad de los frutos.

El uso del Nitrato de potasio (KNO3) es especialmente deseable en condiciones donde se necesita una fuente de nutrientes altamente soluble y libre de cloro. Todo el nitrógeno $(\mathrm{N})$ está inmediatamente disponible para la absorción de las plantas como nitrato, no requiriendo acción microbiana o transformación adicional en el suelo. Los productores de cultivos de hortalizas $\mathrm{y}$ frutales de alto valor a veces prefieren utilizar una fuente nutricional a base de nitrato en un esfuerzo por incrementar rendimiento y calidad. El nitrato de potasio contiene una proporción relativamente alta de $\mathrm{K}$, con una relación $\mathrm{N}: \mathrm{K}$ de aproximadamente 1:3. Muchos cultivos poseen altas demandas de $\mathrm{K}$ y a la cosecha pueden remover tanto o más K que N (IPNI, 2014).

Según datos de la CMB (2013), el Muriato de Potasio, es la fuente de fertilización de Potasio más usada en el mundo. El contenido de Potasio se expresa como equivalente de K2O (Óxido de Potasio) o Potasa. El Cloruro de Potasio $(\mathrm{KCl})$ o Muriato de Potasio (MOP) por su alta concentración de Potasio (60\%) es la fuente de aporte de Potasio (K2O) más económica para la mayoría de los cultivos.

El Sulfato de potasio (K2SO4) es una excelente fuente para la nutrición de las plantas. La porción potásica del K2SO4 no es diferente a la de otras fuentes de fertilizantes potásicos. Sin embargo también aporta una fuente valiosa de azufre (S), que es a veces deficiente para el crecimiento vegetal. El azufre es requerido para la síntesis de proteínas y el funcionamiento enzimático. Hay ciertos suelos y cultivos donde la aplicación de $\mathrm{Cl}$ - debe ser restringida. En estos casos, el K2SO4 es una fuente de $\mathrm{K}$ muy aconsejable. El $\mathrm{K} 2 \mathrm{SO} 4$ posee solo un tercio de la solubilidad del $\mathrm{KCl}$, por lo que no es comúnmente disuelto para la aplicación a través de agua de riego a menos que haya necesidad de aportar S (IPNI, 2014).

Lovatt y Mikkelsen (2006) manifiestan que el fósforo es un elemento esencial requerido por todos los organismos vivos. En forma elemental no aparece en la naturaleza porque es muy reactivo, se combina rápidamente con otros elementos como oxigeno $(\mathrm{O})$ e hidrogeno $(\mathrm{H})$. Cuando se oxida 
completamente, el fósforo se une con cuatro átomos de $\mathrm{O}$ para formar la conocida molécula de fosfato. Sin embargo, cuando no se oxida completamente un átomo de $\mathrm{H}$ ocupa el lugar de $\mathrm{O}$ y la molécula resultante se denomina fosfito. Este aparentemente simple cambio en la estructura molecular causa diferencias significativas que influencian la solubilidad relativa de la molécula y afecta la absorción y metabolismo de las plantas.

Los fosfitos son derivados del ácido fosforoso que se combinan con diferentes elementos como $\mathrm{Ca}, \mathrm{K}, \mathrm{Al}, \mathrm{Mn}, \mathrm{Mg}$ y $\mathrm{Zn}$. Su acción está referida como fertilizante, bioestimulante, inductor de resistencia y en algunos casos, como fungicida tanto en cultivos extensivos como intensivos. Los fosfitos con el catión unido tienen influencia sobre la nutrición de las plantas, entre los cationes principales están potasio, cobre y manganeso. Por eso en algunos cultivos y en dosis adecuadas, el fosfito de potasio incrementa el rendimiento (Carmona y Sautua, 2010).

Para Lovatt y Mikkelsen (1994) el ácido fosforoso (H3PO3) y su sal (fosfito) contiene concentraciones de P (39\%) más altas que los fertilizantes fosfatados (32\%) basados en ácido fosfórico (H3PO4). Las sales de fosfito son generalmente más solubles que las sales análogas de fosfato. El fosfato completamente oxidado es la forma más estable de $\mathrm{P}$ en el ambiente, por esta razón, el fosfito pasa por una transformación gradual después de adicionarse al suelo hasta formar fosfato.

Según NUTRAFEED (2014), los fosfitos actúan estimulando el metabolismo de la planta y equilibra sus funciones fisiológicas a nivel celular, de manera integral desarrolla su potencial productivo frente al estrés climático. Este efecto se traduce en un mejor crecimiento vegetativo, adecuado desarrollo de raíz, floración, fructificación y desarrollo del fruto.

En base a las razones antes mencionadas la investigación buscó: Evaluar el comportamiento agronómico del cultivo a la aplicación de fertilizantes potásicos, Estudiar los efectos de los activadores fisiológicos fosfatados sobre la fisiología de la planta y determinar los tratamientos e interacciones más influyentes sobre el rendimiento del maíz en la región.

\section{Materiales y métodos}

La investigación se efectuó en los terrenos del Señor Santiago Lamilla, ubicados en la parroquia San Juan a $3 \mathrm{~km}$ de la cabecera parroquial. La zona presenta un clima tropical húmedo, está ubicada entre las coordenadas geográficas $79^{\circ} 30^{\prime}$ de longitud occidental y $1^{\circ} 48^{\prime}$ de latitud sur, precipitación promedio de $1980 \mathrm{~mm}$ y temperatura de $25,4{ }^{\circ} \mathrm{C}$. (INAHMI, 2016). El suelo es de origen aluvial, con topografía plana, textura franco arcillosa, drenaje natural. 
Se utilizó como material vegetativo los híbridos de maíz DK-7088 (Ecuaquimica, 2015). En los tratamientos potásicos se realizaron aplicaciones a los 10-20 y 35 días después de la siembra, los fosfitos se aplicaron vía foliar en las mismas fechas.

\section{Tratamientos}

Los tratamientos estuvieron formados por tres fuentes de potasio (Sulfato de potasio, Muriato de potasio y Nitrato de potasio) y tres activadores fisiológicos (Fosfito de potasio, Fosfito de calcio y Fosfito de magnesio), en dosis alta $1 \mathrm{~L} \mathrm{ha-}^{1}$ y baja $0,5 \mathrm{~L} \mathrm{ha-}{ }^{1}$.

Tabla 1. Tratamientos aplicados, Babahoyo, 2016.

\begin{tabular}{|c|c|c|c|c|}
\hline & Fuente de potasio & $\begin{array}{c}\text { Dosis } \\
\left(\mathrm{kg} \mathrm{ha-}^{-1}\right)\end{array}$ & Activador fisiológico & $\begin{array}{c}\text { Dosis } \\
\left(\mathrm{L} \mathrm{ha-}^{-1}\right)\end{array}$ \\
\hline 1 & \multirow{6}{*}{ Muriato de potasio } & \multirow{6}{*}{ 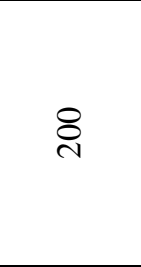 } & Fosfito de potasio & 0,5 \\
\hline 2 & & & Fosfito de calcio & 0,5 \\
\hline 3 & & & Fosfito de magnesio & 0,5 \\
\hline 4 & & & Fosfito de potasio & 1,0 \\
\hline 5 & & & Fosfito de calcio & 1,0 \\
\hline 6 & & & Fosfito de magnesio & 1,0 \\
\hline 7 & \multirow{6}{*}{ Sulfato de potasio } & \multirow{6}{*}{$\stackrel{\text { 工 }}{ }$} & Fosfito de potasio & 0,5 \\
\hline 8 & & & Fosfito de calcio & 0,5 \\
\hline 9 & & & Fosfito de magnesio & 0,5 \\
\hline 10 & & & Fosfito de potasio & 1,0 \\
\hline 11 & & & Fosfito de calcio & 1,0 \\
\hline 12 & & & Fosfito de magnesio & 1,0 \\
\hline 13 & \multirow{6}{*}{ Nitrato de potasio } & \multirow{6}{*}{$\stackrel{\ominus}{=}$} & Fosfito de potasio & 0,5 \\
\hline 14 & & & Fosfito de calcio & 0,5 \\
\hline 15 & & & Fosfito de magnesio & 0,5 \\
\hline 16 & & & Fosfito de potasio & 1,0 \\
\hline 17 & & & Fosfito de calcio & 1,0 \\
\hline 18 & & & Fosfito de magnesio & 1,0 \\
\hline 19 & Testigo agricultor & ---------- & Sin activador fisiológico & --- \\
\hline
\end{tabular}

Para el desarrollo y evaluación estadística del ensayo se utilizó el diseño "Bloques Completo al Azar" (BCA) en arreglo factorial 3x3x2 con 18 tratamientos más un testigo y 3 repeticiones. La comparación y evaluación de las medias se realizó con la prueba de Tukey al $95 \%$ de probabilidad. Se evaluó: altura de planta, días a floración, longitud de mazorcas y rendimiento por hectárea.

Previo al establecimiento del ensayo se tomaron muestras de suelos, para proceder al análisis físico y químico; determinando así la cantidad de nutrientes existentes. El terreno fue preparado con un pase de arado y dos pases de rastra liviana cruzados. 
El cultivo se sembró en hileras a un distanciamiento de $0,8 \mathrm{~m} \times 0,2 \mathrm{~m}$, en parcelas rectangulares $3 \times 4 \mathrm{~m}$ aplicando riego a los 45 días después de la siembra. Las variables agronómicas se evaluaron según criterios técnicos. El control de gusano cogollero (Spodoptera frujiperda) se efectuó con Clorpirifos $\left(0,8 \mathrm{~L} \mathrm{ha}^{-1}\right)$ a los 20 días después de la siembra y a 42 días después de la siembra con Pirimifos+Deltametrina $\left(0,5+0,3 \mathrm{~L} \mathrm{ha}^{-1}\right)$. Se aplicó Diazinon $1 \mathrm{~L} \mathrm{ha}^{-1}$ para el control del insecto chupador Dalbolus, mientras el control de mancha de asfalto (Monographella y Phyllacora) se realizó con Amistar top $\left(0,3 \mathrm{~L} \mathrm{ha}^{-1}\right)$ a los 50 días después de la siembra. Las malezas se controlaron en preemergencia con Pendimentalin $3 \mathrm{~L} \mathrm{ha}^{-1}$, Amina $0,5 \mathrm{~L} \mathrm{ha}^{-1} \mathrm{y}$ Atrazina $1 \mathrm{~kg} \mathrm{ha}^{-1}$, aplicándose a los 35 días después de la siembra $1,0 \mathrm{~L} \mathrm{ha}^{-1}$ de Paraquat.

El cultivo fue realizado en la época lluviosa 2016, con lo cual no se realizó aportes hídricos al ensayo.

Para la aplicación de los activadores fisiológicos, se empleó una bomba de mochila con boquilla de cono sólido. El nitrógeno se aplicó como Urea en dosis de $300 \mathrm{~kg} \mathrm{ha}^{-}{ }^{1}$ a los 10, 20 y 35 días después de la siembra en partes iguales. La aplicación de azufre se realizó utilizando Sulfato de amonio (50 kg ha- ${ }^{1}$ ) a los 15 y 45 días después de la siembra, fraccionando la aplicación en dos partes. Para la aplicación del potasio los diferentes fertilizantes potásicos (Muriato de potasio, Sulfato de potasio y Nitrato de potasio), se fraccionaron en dosis iguales en cada aplicación. El fósforo DAP (80 kg ha- ${ }^{1}$ ), se puso a los 15 días después de la siembra. La aplicación de microelementos se realizó a los 20 días después de la siembra de manera foliar con una bomba de aspersión calibrada y para una mejor eficiencia se utilizó una boquilla de cono sólido.

La altura de planta fue medida con un flexómetro a la cosecha, en 10 plantas al azar por tratamiento desde el nivel del suelo hasta la última hoja emergida, expresando el valor en centímetros.

Los días a floración fueron tomados desde el inicio de la siembra hasta cuando el cultivo tuvo un $70 \%$ de inflorescencias emergidas en cada parcela experimental, se cogió en 10 plantas al azar por tratamiento.

La longitud y diámetro de mazorcas, se midieron en el tercio medio utilizando un calibrador y desde la base o pedúnculo hasta el ápice de la misma, registrando en centímetros, usando una cinta flexible en 10 mazorcas al azar.

El rendimiento por hectárea fue evaluado en cada unidad experimental recolectando la cosecha del área útil y uniformizando al 13\% de humedad. 


\section{Resultados}

\section{Altura de planta}

Con la utilización de fertilizantes potásicos destacó Muriato de potasio con $237,23 \mathrm{~cm}$. En cuanto a los activadores fosfatados, la aplicación de fosfito de calcio $0,5 \mathrm{~L} \mathrm{ha}^{-1}$ obtuvo el mayor valor con $239,62 \mathrm{~cm}$. En las interacciones, el mayor valor lo representaron las plantas fertlizadas con Muriato de potasio utilizando fosfito de calcio $0,5 \mathrm{~L} \mathrm{ha}^{-1}$ con $247,93 \mathrm{~cm}$, estadísticamente igual Muriato de potasio con fosfito de magnesio $0,5 \mathrm{~L} \mathrm{ha}^{-1}(241,20 \mathrm{~cm})$ y Sulfato de potasio con fosfito de potasio con $240,53 \mathrm{~cm}$, superiores estadísticamente al resto de tratamientos, siendo el menor valor para Nitrato de potasio con fosfito de magnesio $0,5 \mathrm{~L} \mathrm{ha}^{-}{ }^{1}$ con $226,73 \mathrm{~cm}$ (Tabla 2).

Tabla 2. Altura de planta de maíz con la aplicación fertilizantes potásicos y fosfitos.

Babahoyo, 2016. (cm)

\begin{tabular}{|c|c|c|c|c|c|}
\hline \multirow[b]{2}{*}{ Fosfitos } & \multicolumn{5}{|c|}{ Fertilizantes Potásicos } \\
\hline & $\begin{array}{c}\text { Muriato } \\
\text { de } \\
\text { Potasio }\end{array}$ & $\begin{array}{c}\text { Sulfato } \\
\text { de } \\
\text { Potasio } \\
\end{array}$ & $\begin{array}{l}\text { Nitrato } \\
\text { de } \\
\text { Potasio }\end{array}$ & Testigo & $\begin{array}{c}\text { Promedio } \\
\mathrm{cm}(\mathrm{ns})\end{array}$ \\
\hline Fosfito de Potasio $0,5 \mathrm{~L} \mathrm{ha}^{-1}$ & 236,87 & 240,53 & 232,93 & & 236,78 \\
\hline Fosfito de Calcio $0,5 \mathrm{~L} \mathrm{ha}^{-1}$ & 247,93 & 235,33 & 235,60 & & 239,62 \\
\hline Fosfito de Magnesio $0,5 \mathrm{~L} \mathrm{ha}^{-1}$ & 241,20 & 227,40 & 226,73 & & 231,78 \\
\hline Fosfito de Potasio 1,5 $\mathrm{L} \mathrm{ha}^{-1}$ & 235,80 & 233,73 & 230,60 & & 233,38 \\
\hline Fosfito de Calcio 1,5 $\mathrm{L} \mathrm{ha}^{-1}$ & 231,40 & 236,00 & 230,07 & & 232,49 \\
\hline Fosfito de Magnesio 1,0 $\mathrm{L} \mathrm{ha}^{-1}$ & 230,20 & 238,47 & 234,53 & & 234,40 \\
\hline Promedio cm (ns) & 237,23 & 235,24 & 231,74 & 228,67 & \\
\hline Coeficiente de variación (\%) & & & 1,64 & & \\
\hline
\end{tabular}

Medias con una letra en común no son significativamente diferentes Tukey $(\mathrm{p} \leq 0,05)$.

\section{Días a floración}

Los tratamientos que tardaron en florecer ( 51,58 y 52,86 días) fueron donde se aplicó Sulfato de potasio y Nitrato de potasio. En las interacciones fueron las aplicaciones de Muriato de potasio + Fosfito de potasio 0,5 L ha (59,50 días) y Muriato de potasio + Fosfito de calcio 0,5 $\mathrm{L} \mathrm{ha}^{-1}$ (58,67 días) las que florecieron tardíamente, mientras con floración precoz las plantas tradas con Sulfato de potasio + Fosfito de calcio $0,5 \mathrm{~L} \mathrm{ha}^{-1}$ (48,67 días) y Nitrato de potasio + Fosfito de potasio 0,5 $\mathrm{L} \mathrm{ha}^{-1}$ (48,00 días) (Tabla 3).

Tabla 3. Días a la floración con la aplicación fertilizantes potásicos y fosfitos. Babahoyo, 2016. (Días)

\begin{tabular}{cccccc}
\hline & \multicolumn{5}{c}{ Fertilizantes Potásicos } \\
\cline { 2 - 5 } Fosfitos & Muriato & Sulfato & Nitrato & \\
& de & de & de & Testigo & Promedio \\
& Potasio & Potasio & Potasio & & (ns) \\
\hline
\end{tabular}




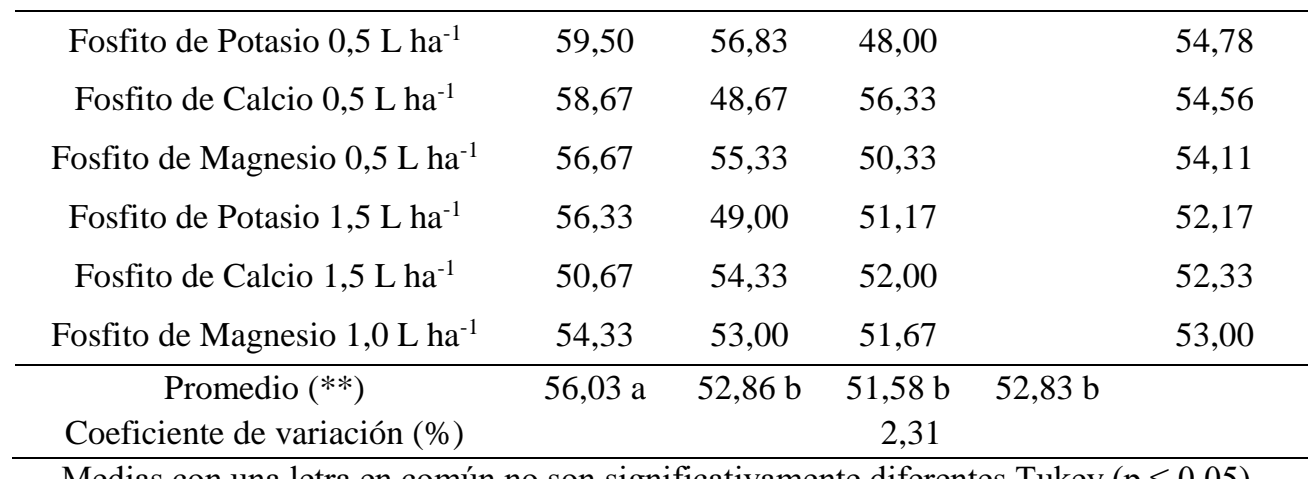

Medias con una letra en común no son significativamente diferentes Tukey $(\mathrm{p} \leq 0,05)$.

\section{Longitud de mazorca}

La aplicación de Nitrato de potasio registró mayor longitud de mazorca $(158,09 \mathrm{~mm})$. El uso de fosfito de magnesio $1,0 \mathrm{~L}$ ha- ${ }^{1}$ presentó mayor diámetro $(161,51 \mathrm{~mm})$. En las interacciones sobresalieron las plantas fertilizadas con Nitrato de potasio más Fosfito de magnesio $1,0 \mathrm{~L} \mathrm{ha}^{-1}{ }^{1}(162,2$ $\mathrm{mm}$ ) (Tabla 4).

Tabla 4. Longitud de mazorca con la aplicación fertilizantes potásicos y fosfitos. Babahoyo, 2016. (mm)

\begin{tabular}{|c|c|c|c|c|c|}
\hline \multirow[b]{2}{*}{ Fosfitos } & \multicolumn{5}{|c|}{ Fertilizantes Potásicos } \\
\hline & $\begin{array}{c}\text { Muriato } \\
\text { de } \\
\text { Potasio }\end{array}$ & $\begin{array}{c}\text { Sulfato } \\
\text { de } \\
\text { Potasio }\end{array}$ & $\begin{array}{c}\text { Nitrato } \\
\text { de } \\
\text { Potasio }\end{array}$ & Testigo & $\begin{array}{l}\text { Promedios } \\
\text { (ns) }\end{array}$ \\
\hline Fosfito de Potasio 0,5 L ha- ${ }^{1}$ & 156,33 & 161,13 & 153,47 & & 156,98 \\
\hline Fosfito de Calcio $0,5 \mathrm{~L} \mathrm{ha-}^{1}$ & 161,47 & 156,13 & 157,93 & & 158,51 \\
\hline Fosfito de Magnesio $0,5 \mathrm{~L} \mathrm{ha-}^{1}$ & 156,40 & 153,87 & 156,13 & & 155,47 \\
\hline Fosfito de Potasio $1,5 \mathrm{~L} \mathrm{ha-}^{1}$ & 150,13 & 159,60 & 159,53 & & 156,42 \\
\hline Fosfito de Calcio $1,5 \mathrm{~L}$ ha- ${ }^{1}$ & 154,00 & 156,27 & 159,07 & & 156,44 \\
\hline Fosfito de Magnesio 1,0 $\mathrm{L} \mathrm{ha-}^{1}$ & 161,07 & 161,07 & 162,40 & & 161,51 \\
\hline Promedio (ns) & 156,57 & 158,01 & 158,09 & 156,80 & \\
\hline Coeficiente de variación (\%) & & & 0,21 & & \\
\hline
\end{tabular}

\section{Diámetro de mazorca}

En diámetro de mazorca las plantas fertilizadas con Nitrato de potasio tuvieron mayor diámetro con $51,83 \mathrm{~mm}$. En activadores fosfatados la aplicación de Fosfito de magnesio 1,0 L ha- ${ }^{1}$ con $52,13 \mathrm{~mm}$, tuvo mayor diámetro en las mazorcas. En las interacciones las plantas donde se aplicó Nitrato de potasio + Fosfito de potasio $1 \mathrm{~L} \mathrm{ha}^{-1}(52,67 \mathrm{~mm})$ presentaron el mayor diámetro (Tabla 5). 
Tabla 5. Diámetro de mazorca con la aplicación fertilizantes potásicos y fosfitos. Babahoyo, 2016. (mm)

\begin{tabular}{|c|c|c|c|c|c|}
\hline \multirow[b]{2}{*}{ Fosfitos } & \multicolumn{5}{|c|}{ Fertilizantes Potásicos } \\
\hline & $\begin{array}{c}\text { Muriato } \\
\text { de } \\
\text { Potasio }\end{array}$ & $\begin{array}{c}\text { Sulfato } \\
\text { de } \\
\text { Potasio }\end{array}$ & $\begin{array}{l}\text { Nitrato } \\
\text { de } \\
\text { Potasio }\end{array}$ & Testigo & $\begin{array}{c}\text { Promedio } \\
\mathrm{mm}(\mathrm{ns})\end{array}$ \\
\hline Fosfito de Potasio 0,5 L ha- ${ }^{1}$ & 51,60 & 52,20 & 51,53 & & 51,78 \\
\hline Fosfito de Calcio 0,5 $\mathrm{L} \mathrm{ha}^{-1}$ & 52,33 & 51,13 & 52,13 & & 51,87 \\
\hline Fosfito de Magnesio $0,5 \mathrm{~L} \mathrm{ha-}^{1}$ & 51,73 & 51,47 & 51,33 & & 51,51 \\
\hline Fosfito de Potasio $1,5 \mathrm{~L} \mathrm{ha-}^{1}$ & 51,00 & 52,07 & 52,67 & & 51,91 \\
\hline Fosfito de Calcio 1,5 $\mathrm{L} \mathrm{ha-}^{1}$ & 51,07 & 51,67 & 51,47 & & 51,40 \\
\hline Fosfito de Magnesio 1,0 $\mathrm{L} \mathrm{ha-}^{1}$ & 52,13 & 52,40 & 51,87 & & 52,13 \\
\hline Promedio mm (ns) & 51,64 & 51,82 & 51,83 & 50,87 & \\
\hline Coeficiente de variación (\%) & & & 0,37 & & \\
\hline
\end{tabular}

Medias con una letra en común no son significativamente diferentes Tukey $(\mathrm{p} \leq 0,05)$.

\section{Rendimiento por hectárea}

La aplicacion de Muriato de potasio reportó mayor rendimiento $\left(8200,82 \mathrm{~kg} \mathrm{ha-}{ }^{-1}\right)$ con relación a los demás fertilizantes, siendo estadísticamente superior a estos. Las plantas tratadsa con Fosfito de Calcio en dosis de 0,5 y $1,0 \mathrm{~L} \mathrm{ha-}^{1}$ registraron más ioncremento en la producción (8340,11 y 8383,28 $\mathrm{kg} \mathrm{ha}^{-}{ }^{-}$, respectivamente), superior estadísticamente a las demás tratamientos. En las interacciones con la aplicacion de Muriato de potasio con Fosfito de potasio 1,0 L ha- ${ }^{1}$ y Muriato de potasio con Fosfito de calcio 1,0 L ha- ${ }^{1}$, se determinó el mayor rendimiento, siendo estas plantas superiores estadísticamente al resto de interacciones, presentando el menor rendimiento $\left(6339,49 \mathrm{~kg} \mathrm{ha}^{-}{ }^{1}\right.$ ) el testigo (Tabla 6).

Tabla 6. Rendimiento por hectárea con la aplicación fertilizantes potásicos y fosfitos. Babahoyo, 2016. (kg ha- ${ }^{1}$ )

\begin{tabular}{|c|c|c|c|c|c|}
\hline \multirow[b]{2}{*}{ Fosfitos } & \multicolumn{5}{|c|}{ Fertilizantes Potásicos } \\
\hline & $\begin{array}{c}\text { Muriato } \\
\text { de } \\
\text { Potasio }\end{array}$ & $\begin{array}{l}\text { Sulfato } \\
\text { de } \\
\text { Potasio }\end{array}$ & $\begin{array}{c}\text { Nitrato } \\
\text { de } \\
\text { Potasio }\end{array}$ & Testigo & $\begin{array}{c}\text { Promedio } \\
\mathrm{s} \\
\mathrm{kg} / \mathrm{ha}^{1}{ }^{1} \\
(* *)\end{array}$ \\
\hline Fosfito de Potasio 0,5 $\mathrm{L} \mathrm{ha-}^{1}$ & 7789,89 & 7566,04 & $\begin{array}{c}7893,0 \\
?\end{array}$ & & $\begin{array}{c}7749,65 \\
\text {. }\end{array}$ \\
\hline Fosfito de Calcio $0,5 \mathrm{~L} \mathrm{ha-}^{1}$ & 8606,66 & 7924,01 & 8489,6 & & $8340,11 \mathrm{a}$ \\
\hline Fosfito de $\underset{1}{\text { Magnesio } 0,5 \mathrm{~L} \text { ha- }}$ & 7324,61 & 7964,71 & 7751,5 & & $7680,28 \mathrm{c}$ \\
\hline Fosfito de Potasio $1,5 \mathrm{~L} \mathrm{ha-}^{1}$ & 8570,13 & 7213,15 & $\begin{array}{c}8000,3 \\
2\end{array}$ & & $7927,87 \mathrm{~b}$ \\
\hline Fosfito de Calcio $1,5 \mathrm{~L} \mathrm{ha-}^{1}$ & 9451,65 & 7961,48 & $\begin{array}{c}7736,7 \\
n\end{array}$ & & 8383,28 a \\
\hline
\end{tabular}




\begin{tabular}{|c|c|c|c|c|}
\hline Fosfito de Magnesio 1,0 L ha- & 7461,98 & 7991,54 & 8186,2 & \\
\hline Promedio kg/ha $(* *)$ & 8200,82 & $\begin{array}{c}7770,15 \\
\text { ?. }\end{array}$ & $\begin{array}{c}8009,5 \\
0-1\end{array}$ & $\begin{array}{c}6339,4 \\
n\end{array}$ \\
\hline Coeficiente de variación (\%) & & & 0,21 & \\
\hline
\end{tabular}

\section{Conclusiones}

Con los resultados obtenidos en el presente trabajo se determinó que la aplicación de fertilizantes a base potasio más la aplicación de activadores fisiológicos fosfatados en diferentes dosis, elevan el rendimiento de granos en el cultivo de maíz de secano. Con las aplicaciones de Muriato de potasio sobre el híbrido de maíz DEKALB-7088, se encontró alta influencia significativa sobre los factores agronómicos de crecimiento, especialmente aquellos relacionados con el rendimiento y producción, manifestando diferencias notables con la no aplicación del mismo. Los rendimientos presentados fueron superiores a los registrados en otros ensayos y en la zona de estudio. Los rendimientos alcanzados para el hibrido DEKALB-7088 con la aplicación de Muriato de potasio con Fosfito de calcio en dosis de 1,0 L ha- ${ }^{1}(9451,65 \mathrm{~kg}$ ha- ${ }^{1}$ ), superan considerablemente la producción de los demás tratamientos.

\section{References:}

1. Carmona, M., Sautua, F. 2010. Impacto de la nutrición y de fosfitos en el manejo de enfermedades en cultivos extensivos de la región pampeana. Universidad de Buenos Aires, Facultad de AgronomíaFAUBA. Manual de cátedra de Fitopatología. Buenos Aires, Argentina. p 19. Disponible en https://www.ipni.net/ppiweb/ CARMONA\%20\%20Nutrici\%C3\%B3n,\%20fosfitos\%20y\%20enfer medades\%20en\%20cultivos\%20extensivos.pdf

2. Corporación Minera de Bolivia (CMB). 2013. Cloruro de Potasio. Ficha Técnica. Bolivia, p: 1-5. Disponible en http://www.evaporiticos.gob.bo/wp-content/uploads/2013/05/TDR KCl.pdf.

3. Departamento de agricultura (USDA). 2004. Manual técnico de fertilización. Comisión de aplicación de fertilizantes. Informe 245. p: 42-65.

4. Ecuaquímica. (2015). "Híbrido DK-7088". In EDIFARM. Vademécum Agrícola 2014, 15ta edición. Editorial Poligráfica, Guayaquil-Ecuador. pp 267-268.

5. García, F. 2013. Más allá de la próxima cosecha: La nutrición de los cultivos en una agricultura sustentable. Instituto Nacional de Tecnología Agropecuaria (INTA) - International Plant Nutrition Institute (IPNI Cono Sur). In Memorias: Jornadas Argentinas de Conservación de Suelos. 2014 (2):1-38. Disponible en 
http://lacs.ipni.net/pdf.

6. Instituto Nacional de Estadística y censos (INEC)-Sistema de Información Nacional de Agricultura, Ganadería y Pesca (SINAGAP). 2014., Sistemas, superficie producción y rendimiento.: cultivo maíz. Disponible en www.agricultura.gob.ec/index.ohp/site-map/2produción. (Último acceso: 13 de abril 2016).

7. Instituto Nacional de Hidrología y Meteorología (INAHMI). (2016). Información climática para Ecuador: Año 2015. Informe Técnico anual. Quito, EC. Disponible http://www.inahmi.gob.ec/estamet.mediaanual.pdf

8. International Plant Nutrition Institute (IPNI). 2014. Fuentes de Nutrientes Específicas. Edición No 11. EE.UU, p 1. Disponible en http://www.ipni.net/publication/nsses.nsf/pdf

9. Lovatt, C.,Mikkelsen, R. 2006. Phosphite fertilizers: what are they? Can you use them? What can the do? Informaciones Agronómicas 3:9-13. Disponible en http://www.ipni.net/publication/ia-lahp.nsf /Fosfito.pdf

10. Ministerio de Agricultura, Ganadería, Acuacultura y Pesca (MAGAP). 2016. Zonificación agroecológica económica del cultivo de maíz duro (Zea mays) en el ecuador. Resumen Técnico, PDF. Quito, EC. 14 p.

11. Melgar, R. 2009. Capítulo V, Potasio, Argentina. p: 1-15, Disponible en http://www.unsam.edu.ar/institutos/centro_ceps/investigaciones/ fertilizantes/capitulo4.pdf

12. Nutrafeed. 2014. Ficha técnica. Fertilizantes Foliares y de Suelo de Alta Eficiencia. Chile, p 2. Disponible en http://terravitaeagro.com/ hojas-tecnicas/inductores-resistencia adquirida/Fosfito40-20.pdf

13. Kant, S., Kafkafi, U. 2010. University of Jerusalem. Food and Environmental Quality Sciences, Rehovot: Absorción de potasio por los cultivos en distintos estadios fisiológicos. University of Jerusalem, Faculty of Agricultural. Resultados de Investigación, Jerusalén-Israel. p 14 (2):264. Disponible en http://www.ipipotash.org/udocs/ Sesion\%20V.pdf 